REGARDS

SUR L'ECONOMIE ALLEMANDE

BULLETIN ECONOMIQUE DU CIRAC
Regards sur l'économie allemande

Bulletin économique du CIRAC

$103 \mid 2011$

Varia

\title{
Recherche appliquée en RFA. Société Fraunhofer : la R\&D au service de l'industrie
}

\section{Andrea Zenker et Volker Tippmann}

Traducteur : Isabelle Bourgeois et Solène Hazouard

\section{OpenEdition}

\section{Journals}

Édition électronique

URL : http://journals.openedition.org/rea/4356

DOI : $10.4000 /$ rea. 4356

ISBN : 978-2-8218-1136-2

ISSN : 1965-0787

Éditeur

CIRAC

Édition imprimée

Date de publication : 16 décembre 2011

Pagination : 19-28

ISSN : 1156-8992

\section{Référence électronique}

Andrea Zenker et Volker Tippmann, « Recherche appliquée en RFA. Société Fraunhofer : la R\&D au service de l'industrie », Regards sur l'économie allemande [En ligne], 103 | décembre 2011, mis en ligne le 01 décembre 2013, consulté le 01 mai 2019. URL : http://journals.openedition.org/rea/4356 ; DOI : $10.4000 /$ rea. 4356 


\section{Recherche appliquée en RFA. Société Fraunhofer : la R\&D au service de l'industrie}

\section{Andrea Zenker, Volker Tippmann}

Les PME industrielles allemandes sont réputées pour être particulièrement innovantes. Si elles pratiquent souvent la $R \& D$ en interne, elles recourent également aux services des centres de recherche appliquée, parmi lesquels les Instituts Fraunhofer.

Le cas de figure parmi les plus fréquents est alors le suivant: une entreprise technologique a besoin d'une installation d'un type particulier, introuvable sur le marché, mais dont l'utilisation permettrait de générer d'importants gains d'efficience et de conférer à l'entreprise un avantage compétitif considérable. Elle s'adresse dans ce cas à la Société Fraunhofer avec laquelle elle signe un contrat. L'entreprise adresse une demande concrète à l'un des instituts de la Société Fraunhofer, spécialisé dans le domaine afférent, et lui passe commande d'une mission de R\&D industrielle. Cette commande peut porter sur l'analyse des derniers résultats de la recherche fondamentale, la conception et le développement d'un prototype, l'installation et la mise en service de l'équipement chez le client, comme sur des missions de conseil ou de formation. Les termes du contrat sont toujours définis en fonction des besoins concrets de l'entreprise, de même que les divers programmes de travail qui sont ensuite réalisés en partie parallèlement au processus de développement interne à l'entreprise, et en partie en coopération étroite entre cette dernière et l'institut Fraunhofer partenaire.

Expliciter cette coopération entre la Société Fraunhofer et les entreprises est l'objet de la présente contribution. Après la présentation des principales caractéristiques du segment de la recherche appliquée en Allemagne, nous y décrirons l'organisation et le mode de fonctionnement de la Société Fraunhofer, ainsi que son mode de coopération avec l'industrie en Allemagne ; enfin, nous présenterons ses activités hors des frontières, plus particulièrement en France.

\section{Recherche appliquée en Allemagne - recherche pour le compte d'entreprises innovantes -}

Les entreprises allemandes sont intensives en recherche et orientées innovation : en 2009, leurs dépenses de R\&D ont frisé les 56 milliards $€$, dont plus de $80 \%$ étaient consacrés à la R\&D en interne. Les quelque $20 \%$ restants étaient affectés à la recherche sur contrats en externe (Stifterverband für die Deutsche Wissenschaft, 2011). A relever aussi que, en comparaison internationale, cet effort d'innovation est largement partagé par les entreprises allemandes, un grand nombre des entreprises de toute taille - y compris les PME - menant des activités liées à l'innovation (Stifterverband für die Deutsche Wissenschaft, 2011 ; Rammer/Pesau, 2011, Commission européenne, 2009). Ces dernières années, les entreprises ont intensifié leur effort de R\&D; leurs dépenses ne représentaient encore qu'un peu plus de 46 millions € en 2004 (Stifterverband). En 2008, par rapport au PIB, leur apport était de 1,84 \% ; la part des universités et des établissements de recherche publics ne s'élevant respectivement qu'à $0,45 \%$ et $0,37 \%$ du total (ibid.).

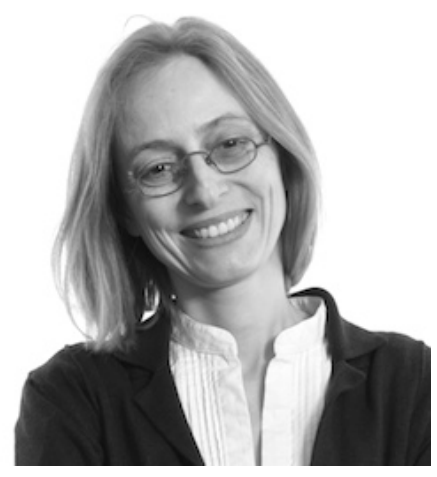

Andrea Zenker,

Responsable de projets au Fraunhofer-Institut für System- und Innovationsforschung

ISI Karlsruhe

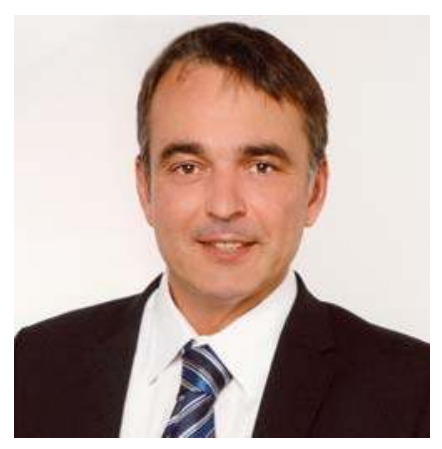

Volker Tippmann, Ingénieur,

Département Planification de la Recherche au siège de la Fraunhofer Gesellschaft, Munich
Entreprises :

2/3 de l'effort de R\&D 
Une structure de la recherche qui favorise le transfert

Une autre caractéristique du système allemand de recherche et d'innovation est l'existence d'un tissu dense et diversifié d'établissements de recherche universitaire et extra-universitaire qui se distingue par un clair partage des rôles et missions entre les diverses sociétés de recherche, ainsi que, en comparaison internationale, l'importance particulière accordée au transfert des savoirs et des technologies. Cet échange entre établissements de recherche et industrie ne favorise pas seulement l'introduction de nouvelles technologies dans les entreprises. Les résultats de la recherche sont au fondement même de l'innovation, et les besoins en matière de recherche orientée vers le marché accroissent dans le même temps les ressources propres (contrats) des établissements de recherche. Le résultat est que, dans les établissements de recherche extra-universitaire, la part de la recherche financée par les entreprises est proportionnellement très élevée. Et souvent, cette coopération entre le secteur de la recherche et les entreprises repose sur des liens établis de longue date. II en va de même des liens étroits de partenariat existant au sein de la recherche universitaire ou entre les établissements de recherche extra-universitaire (Polt et al., 2009 ; Commission européenne, 2009 ; Frietsch/Kroll, 2010).

Les caractéristiques ici esquissées - à savoir une approche de l'innovation profondément ancrée dans la culture économique et la pratique entrepreneuriale allemandes, ainsi qu'un tissu dense et différencié d'acteurs de la recherche sont les deux éléments clés du système d'innovation allemand. Parmi ces acteurs institutionnels de la recherche extra-universitaire se distingue la Société Fraunhofer de promotion de la recherche appliquée (Fraunhofer-Gesellschaft zur Förderung der angewandten Forschung e.V.), un organisme associatif d'intérêt public dont la vocation est la recherche appliquée, proche du marché.

Nous en présentons ci-dessous les activités et l'organisation sous trois principaux angles: quelles sont les missions que poursuivent la Société Fraunhofer et ses Instituts ? Quels sont les mécanismes qui les mènent à poursuivre leurs activités de recherche proches des entreprises ? Et, enfin, comment la Société Fraunhofer se positionne-t-elle dans le contexte européen et international ? Nous nous concentrerons ici sur la coopération avec la France.

\section{Positionnement et mode de fonctionnement du réseau Fraunhofer}

Une recherche extra-universitaire dense et diversifiée

Quatre grands réseaux
En Allemagne, le secteur de la recherche extra-universitaire comprend les Akademien der Wissenschaften qui mènent des études au long cours (par exemple en lexicographie comme la Bayerische Akademie der Wissenschaften ou la Nordrhein-Westfälische Akademie der Wissenschaften und der Künste), les centres de recherche conseillant les ministères techniques du Bund ou des Länder (par exemple : la Bundesanstalt für Geowissenschaften und Rohstoffe ou le Robert-Koch-Institut), les bibliothèques et archives scientifiques, les centres d'information spécialisée (par exemple : Bundesagentur für Außenwirtschaft, Bundesanstalt für Landwirtschaft und Ernährung, Deutsches Institut für Normung e.V.), les musées dotés de missions de R\&D, ainsi que diverses autres institutions de recherche (Polt et al., 2009).

A cela s'ajoute le groupe des quatre grands établissements de recherche extrauniversitaire que sont: la Société Fraunhofer; la Société Max-Planck (MaxPlanck-Gesellschaft e.V.) dont les 80 instituts ont une mission de recherche fondamentale dans les domaines des sciences naturelles, sciences de la vie et sciences sociales; la Communauté Helmholtz (Helmholtz-Gemeinschaft Deutscher Forschungszentren e.V.) et ses 17 centres spécialisés dans la recherche au long cours dans six domaines (énergie, sciences de la terre et de l'environnement, santé, technologies-clés, structure des matériaux et aviation/ espace/transports). Le quatrième est le réseau Leibniz (Wissenschaftsgemeinschaft Gottfried Wilhelm Leibniz e.V.) avec ses 87 établissements («Blaue 
Liste »), et qui se concentre sur les grandes questions de société, met à disposition infrastructures scientifiques et prestations de service afférentes, et a pour axes de recherche notamment les sciences de la nature, les sciences de l'ingénieur, les sciences économiques ou sociales.

Répartition de l'effort de R\&D en Allemagne (2009)

\begin{tabular}{|c|c|c|c|}
\hline & $\begin{array}{l}\text { Dépenses } \\
\text { (en millions } € \text { ) }\end{array}$ & $\begin{array}{c}\text { Effectifs de R\&D } \\
\text { (équivalent temps plein) }\end{array}$ & $\begin{array}{l}\text { dont } \\
\text { scientifiques }\end{array}$ \\
\hline Secteur public et organisations privées à but non lucratif & 9932 & 86633 & 49241 \\
\hline $\begin{array}{l}\text { Ets. de recherche du Bund, des Länder et des communes } \\
\text { (activités de R\&D), hors Communauté Leibniz }\end{array}$ & 1117 & 11695 & 5476 \\
\hline $\begin{array}{l}\text { Ets. de recherche à financement majoritairement public } \\
\text { (activités de R\&D) : Helmholtz, Max-Planck, Fraunhofer, } \\
\text { Leibniz, autres, bibliothèques scientifiques et musées }\end{array}$ & 8815 & 74937 & 43764 \\
\hline Universités (activités de recherche) & 11808 & 115441 & 84771 \\
\hline Entreprises & 45275 & 332491 & 183214 \\
\hline Total & 67015 & 534565 & 317226 \\
\hline
\end{tabular}

Source des données: Destatis, Statistisches Jahrbuch 2011. NB : En 2009, les établissements de recherche extra-universitaire réalisaient quelque $15 \%$ des dépenses de R\&D et occupaient plus de $15 \%$ des scientifiques.

Ces quatre organismes se distinguent par leur domaine de recherche comme leurs missions et, sur cette base, par le profil de leurs utilisateurs ou clients, ainsi que par leurs orientations en matière de publications et de brevets, de même que par la nature de leurs fonds propres. Ainsi, par exemple, la Société Max-Planck (recherche fondamentale) est financée à hauteur d'environ $80 \%$ par des fonds publics ; la société Helmholtz à $70 \%$, et la Communauté Leibniz aux deux tiers (voir notamment www.bmbf.de ou Polt et al., 2009). A l'inverse, dans le cas de la Société Fraunhofer, qui est clairement axée sur la recherche appliquée, le transfert des savoirs et des technologies, ainsi que sur le développement des technologies, la part du financement public et institutionnel n'est que de quelque $30 \%$. En comparaison des trois autres grands réseaux d'établissements, les Instituts Fraunhofer, qui recrutent majoritairement leur clientèle parmi les entreprises privées, sont aussi ceux qui déposent le plus grand nombre de brevets. Quant aux Instituts Max-Planck, ils se distinguent par la plus forte intensité des publications (Polt et al., 2009).

La mission centrale dont s'est dotée la Société Fraunhofer réside dans la recherche pour la praxis, c'est-à-dire débouchant sur une application concrète dans l'entreprise ou la sphère publique. Elle entend ainsi contribuer à la capacité d'innovation de l'économie allemande ainsi qu'à la compétitivité du "site Allemagne ». La recherche menée au sein de la Société Fraunhofer est donc axée sur des missions et objectifs spécifiques : elle vise à développer des solutions répondant à des problèmes donnés, particuliers. Pour ce faire, elle recourt aux connaissances issues de la recherche fondamentale et les applique au problème en question afin de développer des solutions concrètes répondant à la demande de ses clients.

Dans la praxis, cela signifie qu'un Institut Fraunhofer réalise des programmes de R\&D pour le compte d'une entreprise industrielle ou de l'administration. Clients ou donneurs d'ordre - souvent d'ailleurs en coopération avec d'autres partenaires issus des milieux économiques et scientifiques - émettent une demande précise d'étude à l'attention des instituts Fraunhofer. De leur côté, les 80 établissements de la Société Fraunhofer, dont ses 60 instituts, proposent, selon la demande et sa spécialisation, une large palette de prestations dans de nombreux domaines de compétences, notamment en matière de santé, sécurité, information et communication, énergie, production, environnement ou mobilité. Bien que le portefeuille technique et scientifique se présente sous une grande hétérogénéité, la procédure concrète de passation de commande est la même pour chaque programme : donneurs d'ordre privés et publics signent un contrat avec la Société Fraunhofer. La recherche sur commande ou contrat représente
Financement essentiellement public pour la recherche fondamentale, privé pour la recherche appliquée

Fraunhofer : mission axée sur la demande des clients

Recherche sur contrat : $70 \%$ de ses recettes 
ainsi $70 \%$ des recettes de ses activités de recherche; à peine $30 \%$ de son budget provient de subventions de base du Bund et/ou des Länder d'implantation des instituts ; celles-ci sont utilisées notamment pour financer la recherche initiale interne.

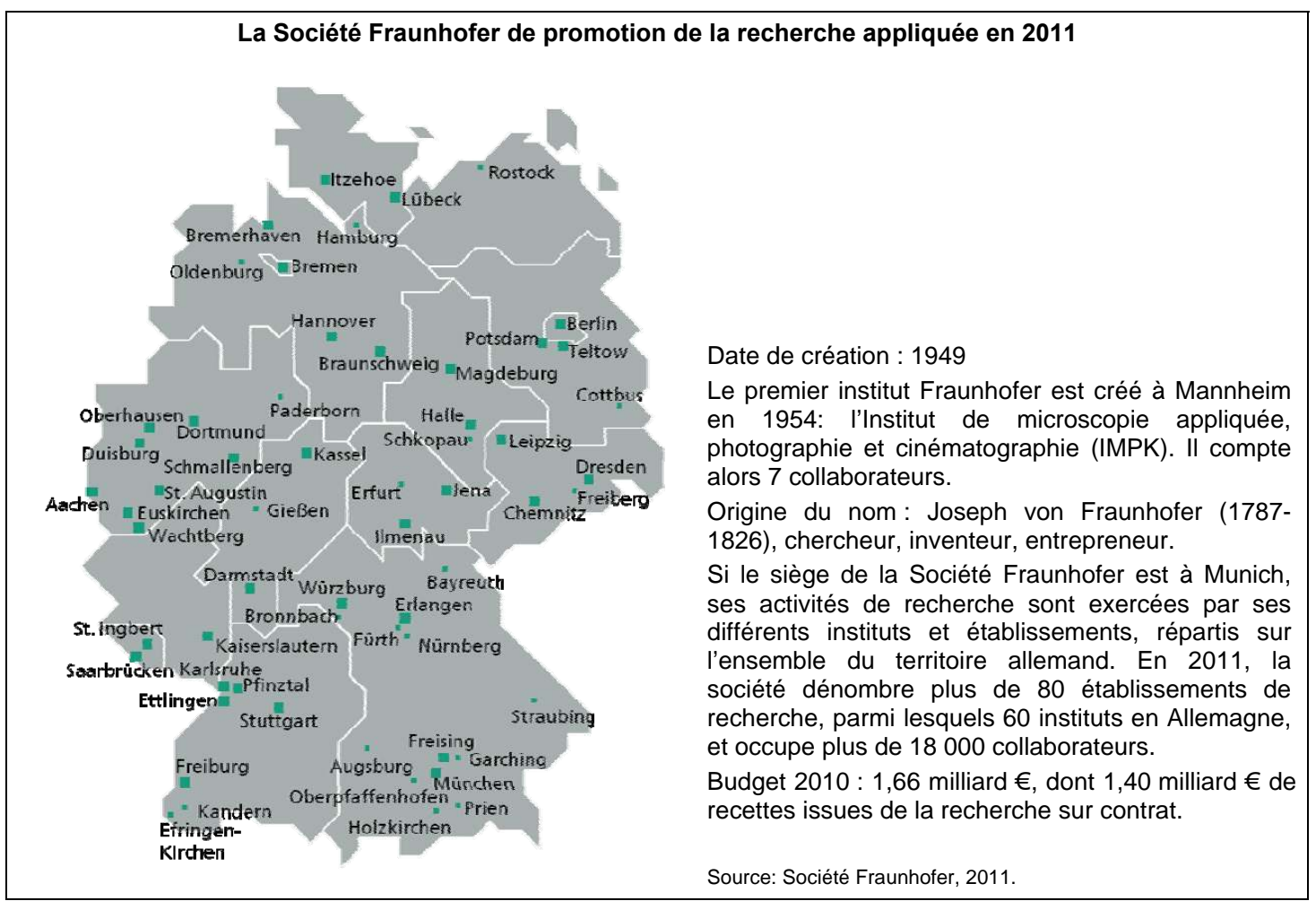

Excellence scientifique et recherche axée sur l'application

Recherche contractuelle orientée projets
De la mission de la Société Fraunhofer découlent certaines spécificités : excellence scientifique et recherche axée sur l'application ; recherche contractuelle orientée projets ; accès à un large éventail de compétences grâce à l'organisation en réseau de la société Fraunhofer.

S'ils sont tributaires de la rentabilité de leurs activités de recherche, les instituts Fraunhofer n'en ont pas moins pour principe de viser l'excellence dans leurs prestations de recherche. Ils développent pour leurs clients des solutions sur mesure qui intègrent aussi les découvertes de la recherche fondamentale et les technologies d'avenir. Cette approche est favorisée par l'implication dans les débats scientifiques en cours et par l'application des découvertes ou solutions ainsi développées aux problèmes concrets qui se posent dans la pratique.

La proximité avec la recherche fondamentale et le développement technologique peut emprunter plusieurs canaux : la coopération avec le secteur universitaire et l'engagement des enseignants-chercheurs (les directeurs d'instituts Fraunhofer occupent la plupart de temps également un poste de professeur d'université et mènent ces deux activités de front), l'encadrement de stages, de mémoires d'études et de thèses de doctorats au sein des instituts Fraunhofer, les publications scientifiques des collaborateurs internes ou encore les séjours de recherche, la participation à des séminaires et conférences, etc. S'y ajoute la recherche initiale menée en interne et financée par les fonds publics, qui permet d'asseoir sur des bases scientifiques la recherche commanditée par les clients et qui entretient la capacité d'innovation de la Société Fraunhofer.

Les travaux de recherche appliquée de la Société Fraunhofer s'effectuent sous forme de programmes ou projets. Un programme ou projet correspond à une mission de recherche venant de l'industrie ou de donneurs d'ordre publics. II est traité par des équipes qui rassemblent des collaborateurs dotés des diverses 
compétences afférentes. Ces équipes interdisciplinaires sont variables et provisoires : elles sont formées ad hoc pour une mission donnée et n'existent que pour la durée du projet. Etant donné que chacun des Instituts Fraunhofer et de ses groupes de travail traite de nombreux projets en parallèle, dont l'échéance et le contenu varient, cela implique pour les personnes concernées de travailler avec des groupes de collègues dont la composition varie elle aussi selon le projet. Lorsque cela se révèle utile au bon déroulement du projet, la formation des équipes s'effectue par-delà les divisions ou départements de chacun des instituts. Cette flexibilité et l'interdisciplinarité qui en résulte permettent de prendre en considération le problème posé sous différents angles, ce qui favorise en outre le transfert de savoirs au sein de l'institut. La réponse aux commandes passées est donc non seulement flexible, mais aussi très rapide, ce qui correspond foncièrement à la demande de l'industrie comme à ses besoins.

Cette flexibilité interne à chaque institut se trouve démultipliée par l'organisation en réseau de la Société Fraunhofer, qui permet de mutualiser les différentes compétences pour un traitement large et interdisciplinaire du problème posé. Ainsi, les instituts spécialisés dans des domaines proches se sont organisés en groupes thématiques (Fraunhofer Verbund) dans différents champs technologiques (technologies de l'information et de la communication, sciences de la vie, lumière et surfaces, microélectronique, recherche en matière de défense et de sécurité, production, ainsi que matériaux et éléments de construction), afin de pouvoir proposer ensemble, sur le marché de la $R \& D$, leurs compétences ainsi optimisées, car conjuguées.

Il en va différemment des Alliances Fraunhofer (Fraunhofer Allianzen) : dans ce cas, instituts ou départements d'instituts aux compétences clés les plus variées s'allient pour travailler en commun sur un domaine d'activité, par exemple la production automobile, le BTP, le cloud computing ou les transports. Une troisième approche est celle des clusters d'innovation pour la mise en réseau des ressources et compétences en vue de l'échange et du transfert des savoirs. Leur particularité est l'intégration de la composante régionale, c'est-à-dire la conjugaison des forces dans un contexte de proximité géographique dans l'objectif de développer les flux de savoir entre acteurs différents pour générer de nouveaux cheminements innovants. Contrairement aux groupes thématiques et aux alliances, les clusters d'innovation ne sont donc pas constitués uniquement d'établissements de la Société Fraunhofer, mais incluent d'autres acteurs régionaux comme des entreprises industrielles et des centres de recherche universitaire et extra-universitaire. Mais au cœur de cette coopération se trouve toujours un projet spécifique (Société Fraunhofer, 2003 et 2011).

\section{Coopération avec l'industrie, promotion des processus innovants dans l'entreprise}

Les entreprises innovantes doivent faire face à la complexité croissante du processus d'innovation comme au raccourcissement des cycles d'innovation. Faire appel à des acteurs et compétences extérieurs à l'entreprise leur permet de mieux affronter ces défis. Et pour ces entreprises, il est souvent plus efficace d'avoir recours à des prestataires spécialisés externes que de développer les capacités de R\&D nécessaires en interne. Elles s'adressent donc à d'autres entreprises, à des prestataires de services spécialisés ou encore à des instituts de recherche universitaire et extra-universitaire, ce qui permet à ces derniers, grâce aux contrats de R\&D ainsi conclus, d'accéder au financement sur projets de la part des entreprises (voir aussi Koschatzky et al. 2003).

Par son offre et la possibilité qui lui est offerte de recourir à de vastes domaines de compétences au sein de son propre réseau, la Société Fraunhofer joue donc un rôle de premier plan dans la maîtrise des enjeux de l'innovation par les entreprises. Car le large éventail des domaines technologiques et des compé-
Accès à un large éventail de compétences et organisation en réseau
Les services demandés par les entreprises 
Financement sur contrats privés : 436 millions $€$ en 2010

Un réseau très prisé des entreprises

Une stratégie d'internationalisation accrue tences, de même que la réactivité comme la flexibilité au sein du réseau permettent à la Société Fraunhofer de traiter rapidement les demandes de ses clients et de leur offrir des solutions, même complexes. Les services recherchés par ses clients, parmi lesquels figurent de nombreuses petites et moyennes entreprises du Mittelstand, comprennent :

- la R\&D : dans leur domaine de compétence respectif, les instituts Fraunhofer élaborent des prestations de R\&D pour le secteur public et privé, avec pour objectif de développer pour leurs clients des solutions pour la praxis (d'ordre technique ou organisationnel par exemple). Ces solutions vont de l'innovation process ou produit à la fabrication de prototypes ;

- les études et analyses : il s'agit essentiellement d'analyses ciblées, par exemple dans le domaine de la faisabilité, de l'acceptation, de l'observation des marchés ou de l'évolution des tendances, des bilans environnementaux, de la rentabilité, des autorisations, des contrôles de produits chimiques, etc. ;

- le conseil et l'accompagnement : ce champ d'activité vise à aider les clients dans la mise en œuvre d'innovations technologiques ou organisationnelles. Les instituts Fraunhofer peuvent expérimenter ces innovations, accompagner les clients et leurs collaborateurs par le biais de formations, proposer d'autres services d'accompagnement ou apporter des conseils sur les programmes publics de soutien à l'innovation ;

- les contrôles et certifications : les instituts Fraunhofer disposent, dans certains domaines technologiques, de structures de mesures, de tests et de contrôle, et ils proposent la réalisation de séries de tests spécialisés, ainsi que divers services de contrôle et l'octroi de labels en la matière.

En 2010, les recettes de la recherche sur contrats (72\% du total) étaient issues à hauteur de $34 \%$ des entreprises, les $38 \%$ restants provenant du secteur public (dont $13 \%$ de donneurs d'ordre étrangers). Si la Société Fraunhofer n'a pas encore retrouvé, après la crise économique, le niveau des financements privés d'avant (il atteignait $39 \%$ en 2006), celui-ci a enregistré tout de même une hausse de $3 \%$ par rapport à 2009. En 2010, il se montait ainsi à 436 millions $€$, dont 93 millions $€$ de royalties.

Du point de vue des entreprises, les critères déterminants pour l'octroi d'une commande à la Société Fraunhofer sont: son savoir-faire, ses compétences, ses ressources et l'éventail de prestations proposées, ainsi que la large gamme de services offerts en matière de process et qui s'étend tout au long de la chaîne, de la recherche fondamentale jusqu'au développement de prototypes. Les clients de la Fraunhofer apprécient en outre sa neutralité et sa fiabilité, sa flexibilité et son expérience, sa proximité avec le monde de l'entreprise et de l'industrie, son professionnalisme et la qualité du travail réalisé.

Les entreprises disposent de nombreuses possibilités pour contacter le réseau Fraunhofer. Par exemple, dans le cadre d'un projet mené pour le compte de la Commission européenne ou d'un autre donneur d'ordre (public), les grands consortiums peuvent faire office d'intermédiaire pour de futurs contrats de recherche bilatéraux. De même, ce rôle de courroie de transmission peut être joué par d'autres entreprises, qui avaient déjà coopéré avec la Société Fraunhofer, et qui diffusent leur expérience auprès de leurs partenaires, ou encore par les personnes et leurs réseaux de contacts humains.

La Société Fraunhofer met elle aussi en œuvre divers outils et mécanismes incitatifs pour favoriser les processus de création et d'échange de savoirs avec les entreprises clientes, développer de nouvelles idées innovantes et soutenir les processus d'innovation. Parmi ces instruments, on trouve la mise en réseau des milieux scientifiques et économiques à l'échelle internationale, le dépôts de brevets et la prise de participations dans des entreprises porteuses d'avenir et des spin-offs.

La Société poursuit l'objectif d'une internationalisation accrue, notamment via des coopérations avec des partenaires scientifiques étrangers (par exemple 
sous la forme de coopérations avec des universités et des centres de recherche, des coopérations scientifiques bilatérales ou dans le cadre de projets européens), ou via l'implantation d'instituts à l'étranger. Ainsi, la Fraunhofer compte actuellement trois filiales aux Etats-Unis, en Autriche et en Tyrol du Sud. Afin de protéger la propriété intellectuelle et d'assurer l'avantage compétitif issu d'une invention, l'activité de dépôt de brevets au sein de la Fraunhofer s'appuie sur des outils comme l'Intellectual Property Management et des institutions comme la fondation Fraunhofer-Zukunftsstiftung, créée en 2008 pour financer la recherche en amont de la constitution ciblée de clusters.

Enfin, la Société a mis en place un programme de soutien aux spin-offs : «FFE - Fraunhofer fördert Existenzgründungen ", incluant financement initial et élaboration du business plan. 15 projets sont ainsi soutenus tous les ans. A cela s'ajoute le soutien au financement de la création d'entreprises apporté par Fraunhofer Venture (www.fraunhoferventure.de). Les 150 spin-offs créées jusqu'à présent offrent en effet une possibilité de transfert de technologies et de savoirs 'par les têtes', c'est-à-dire de diffusion et de distribution des savoirs par le biais des personnes. La mise en œuvre d'idées et de solutions techniques au fondement de la création de ces sociétés est encouragée, d'un côté comme canal de diffusion du savoir vers les entreprises et, de l'autre, en vue d'élargir les réseaux, notamment d'anciens. Ainsi, un programme spécifique d'essaimage doit venir soutenir les collaborateurs de la Fraunhofer dans leur projet de création d'entreprise, la Société Fraunhofer prenant en outre des participations dans la spin-off qui en résulte.

Concernant le développement et la qualification de son personnel, une série de mesures et programmes a été adoptée : encadrement de thèses de doctorat conjointement avec les universités, qualification scientifique et technologique pour l'acquisition, l'exécution et le management de projets et, bien entendu, mesures de formation continue. Pour recruter la relève, la Société mène par ailleurs une stratégie suivie d'information dans les établissements scolaires et universitaires.

La Société Fraunhofer est par nature favorable à la mobilité de ses personnels, que celle-ci poursuive l'objectif $d u$ «transfert des savoir-faire par les têtes », celui d'accroître la dynamique du réseau interne de R\&D ou celui de recourir régulièrement à de nouveaux collaborateurs et à leur savoir, ce qui permet de renforcer aussi les échanges avec les universités (Trischler/ vom Bruch 1999, Société Fraunhofer 2011).

\section{Les innovations transfrontalières, pierre angulaire de la stratégie d'internationalisation de la Société Fraunhofer : le Programme Inter Carnot Fraunhofer (PICF)}

Dans un monde de plus en plus interconnecté, la coopération internationale est indispensable ; la Société Fraunhofer la considère de ce fait comme un élément essentiel pour la création et l'échange des savoirs. Cette internationalisation s'effectue à différents niveaux, et est encouragée par plusieurs moyens. Dans le cas de la coopération avec la France, par exemple, elle prend la forme d'un programme d'aide bilatéral au renforcement de la coopération scientifique franco-allemande.

Depuis la fin des années 1990 et la Loi sur I'Innovation et la Recherche (1999), le système français de recherche et d'innovation subit une profonde mutation, qui s'accompagne de l'apparition de nouveaux acteurs, institutions et structures de pilotage. Entre autres, priorité a été donnée au soutien aux transferts de technologies, à l'utilisation des résultats de la recherche et à l'innovation. La promotion de la recherche appliquée s'est également imposée comme un axe prioritaire. Lors du deuxième forum franco-allemand de la recherche en 2005 avait ainsi été conclu entre le ministère français de la Recherche et la Société
Spin-offs,

transfert des savoirs...

... et promotion des ressources humaines

Mobilité

2005 : création des Instituts Carnot en France 
A partir de 2007 : lancement de programmes de recherche bilatéraux

Mise en réseau pour créer des synergies

Développement de nouveaux modèles de coopération institutionnelle

Elaboration conjointe de nouveaux concepts d'innovation ouverte
Fraunhofer un accord portant sur la création des Instituts Carnot. Pour la R\&D appliquée à l'industrie, ces derniers ont été dotés d'un label de qualité Carnot (limité dans le temps) et se sont vu attribuer des moyens financiers supplémentaires par l'Agence Nationale de la Recherche (ANR).

Pour soutenir la coopération scientifique entre les instituts Carnot et Fraunhofer, l'ANR et le ministère fédéral de l'Education et de la Recherche (BMBF) ont lancé un programme de recherche bilatéral en 2007. Alors qu'il existait, à l'échelon national, nombre de programmes d'aides pour relier les établissements de recherche entre eux, il n'existait jusqu'alors guère de programmes franco-allemands soutenant les coopérations sur projets. Outre l'échange et la mise en réseau des compétences, leur objectif fondamental résidait dans la mise en place à long terme de partenariats stratégiques entre les instituts de recherche des deux pays. Si l'évaluation des propositions au premier appel d'offres en 2007 s'était encore effectuée dans un cadre national, respectivement par l'ANR en France et, en Allemagne, par la Société Fraunhofer en tant que porteur de projet du BMBF, le deuxième appel à projets, organisé et mené de concert en 2009, peut être considéré comme l'un des premiers programmes bilatéraux d'aide à la recherche en Europe. Depuis, deux autres appels à projets ont suivi, en 2010 et 2011 . Cette coopération franco-allemande $(30$ millions $€$, financés à parité) a permis de lancer jusqu'ici 26 projets de coopération.

Ce programme de coopération a pour principal objectif de former des alliances stratégiques entre les partenaires scientifiques, c'est-à-dire de s'orienter vers des objectifs stratégiques communs établis sur le long terme et d'agir ensemble sur les marchés technologiques. L'idée centrale est de mettre en réseau les compétences complémentaires des instituts Carnot et Fraunhofer, afin de générer des effets de synergie qui produiront à leur tour un avantage compétitif technologique dans la R\&D nationale et internationale. Pour la Société Fraunhofer, cela signifie l'élargissement, par-delà les frontières allemandes, de son réseau de partenariats d'excellence ; outre-Rhin, elle travaille avant tout en étroite coopération avec les universités. Cette coopération permet ainsi d'identifier des partenaires qui, dans des domaines complémentaires, font preuve d'excellence, et de construire sur cette base une coopération fructueuse pour les deux parties.

La Société Fraunhofer vise ce faisant à étendre sur le moyen et le long terme hors du territoire un modèle de coopération institutionnelle qui a fait ses preuves en Allemagne, à savoir mettre en place, avec des partenaires français, une coopération institutionnelle sous la forme d'unités de recherche conjointes et d'instituts communs. Le critère décisif est celui de la qualité du partenariat et le succès du travail commun, notamment au regard des résultats exploitables à l'international. Jusqu'à présent, cette forme de coopération de la Société Fraunhofer se limite à quelques rares pays dans le monde. La France présente un intérêt particulier, du fait de ses excellentes organisations de recherche et de son importance en tant que partenaire commercial de l'Allemagne. Dans le cadre de la coopération avec les instituts Carnot seront donc développés de nouveaux modèles de coopération institutionnalisée, susceptibles par la suite d'être appliqués à d'autres pays européens.

Ce programme vise également à promouvoir les transferts de savoirs et de technologies vers les entreprises industrielles, afin d'accroître leurs capacités d'innovation et leur compétitivité. A travers la coopération d'instituts de recherche spécialisés issus de différents contextes nationaux, le socle de savoirs et de compétences des travaux de recherche pourra être considérablement élargi, au profit des clients dans les deux pays. Dans le cadre des projets conjoints sont élaborés de nouveaux concepts d'innovation ouverte (open innovation), c'est-à-dire la mise en œuvre stratégique de moyens d'innovation externe (en plus des moyens internes) à l'entreprise, y compris par exemple des coopérations dans le domaine de la $R \& D$ ou l'intégration de la demande concrète des 
clients. Son objectif est de construire, parallèlement à la coopération purement scientifique, un réseau d'entreprises innovantes, allemandes et françaises.

Le processus de développement de cette stratégie, dont l'accompagnement est financé par le BMBF, vise à élaborer de nouvelles approches et connaissances en matière de conception d'alliances franco-allemandes dans le domaine de la R\&D. Concrètement, la mesure d'accompagnement vise à analyser les conditions nécessaires au lancement et au développement de coopérations stables et durables. L'évaluation des expériences menées jusqu'ici a en effet permis de déceler l'importance du processus apprenant que présente une telle coopération et d'en tirer les conclusions en termes d'apprentissage (learning effects). Entre les partenaires français et allemand, il existe en effet de nombreuses différences quant à la structure des paysages de la recherche respectifs, aux traditions dans la recherche appliquée, aux institutions et enfin aux cultures mêmes du travail. II s'agit dès lors d'identifier ces différences et d'en faire jaillir les complémentarités afin de mieux faire fructifier ces compétences dans l'intérêt conjoint des partenaires (Muller, Zenker, Héraud, 2010 ; Tippmann, Zenker, Muller, 2010).

LA SOCIÉTÉ FRAUNHOFER N'EST PAS LE LIEU DES 'GRANDES RUPTURES', mais celui où naissent toutes ces 'petites ruptures' qui, dans la praxis des entreprises tout particulièrement celles du Mittelstand -, génèrent et entretiennent à la fois ce process de perpétuelle innovation au fondement de leur compétitivité.

Or pour jaillir, ces innovations ont besoin du terreau favorable que seules peuvent créer des relations étroites et surtout établies dans la durée. Seule la continuité des contacts entre personnes et celle des échanges scientifiques et techniques permet de nourrir la confiance, de même que la compréhension intime des possibilités et limites respectives de la coopération - ces conditions préalables indispensables à l'élaboration d'une coopération stratégique. Et c'est seulement quand cette coopération a pris une dimension stratégique commune et partagée que naît ce processus dont jaillissent ces 'petites ruptures' qui, à force de se multiplier, finissent par engendrer parfois d'importantes mutations.

Bien entendu, toutes les coopérations avec les entreprises industrielles ou d'autres établissements de recherche ne mènent pas toujours à cette constellation idéale. Mais l'approche des Instituts Fraunhofer et les instruments développés visent très précisément à faire naître de telles formes de coopération pour établir des passerelles durables entre la recherche fondamentale des universités et la production des entreprises industrielles.

C'est là par exemple l'objectif du Programme Inter Carnot Fraunhofer: il ne s'agit pas seulement de développer un réseau international d'échanges de travaux de recherche, mais avant tout de construire une coopération stratégique établie sur le long terme avec des partenaires choisis pour leur complémentarité. Une bonne illustration en est le projet Solarbond qui associait l'Institut Carnot LETI, l'Institut Fraunhofer ISE et la société Soitec, et qui s'est vu décerner cette année le Prix franco-allemand de l'Economie dans la catégorie Innovation. Inscrire dans la durée un tel partenariat stratégique exige que soient réunies de multiples conditions, ce qui constitue un véritable défi, surtout au niveau international. Le projet Solarbond représente une première étape importante dans la réalisation de cet objectif. II convient maintenant d'établir sur cette base, et pour tous les participants à ce projet, une situation de win-win inscrite dans la durée.

(Traduction : I. Bourgeois et S. Hazouard)

\section{Indications bibliographiques}

-BouRgeols I., "L'atout décisif des PME industrielles : innovation et intelligence de l'économie ", in BOURGEOIS I. (dir), PME allemandes : les clés de la performance, Cergy-Pontoise, 2010

- Bundesministerium für BILdUNg UND Forschung (BMBF), Bundesbericht Forschung und Innovation 2010, Bonn, Berlin, 2010 
- Commission européenne, DiRection Générale ENTREPrise (eds), INNO-Policy TrendChart Innovation Policy Progress Report Germany, 2009. Rapport réalisé par Christian Rammer (ZEW, Mannheim). Consultable sur: www.proinno-europe.eu/page/innovation-and-innovation-policygermany [consulté le 21-09-2011]

-Deutsche Telekom Stiftung, BDI (eds), Innovationsindikator 2011, Bonn/Berlin, 2011. Consultable sur : www.innovationsindikator.de (consulté le 07-12-2011)

- Fraunhofer-Gesellschaft zUR Förderung DER ANGeWANDTEN Forschung E.V., Leitbild der Fraunhofer-Gesellschaft, Munich, 2003. Consultable sur: www.fraunhofer.de/publikationen/ broschueren/Leitbild_Fraunhofer.pdf [consulté le 21-09-2011]

- FRIETSCH R., KROLL H., « Recent Trends in Innovation Policy in Germany », in FRIETSCH R., SCHÜLLER M. (eds), Competing for Global Innovation Leadership : Innovation Systems and Policies in the USA, Europe and Asia, Fraunhofer Institute for Systems and Innovation Research ISI, Stuttgart, 2010

- HazouARd S., LASSerRe R., Uterwedde H. (dir), Les politiques d'innovation coopérative en Allemagne et en France : Expériences et approches comparées, Cergy-Pontoise, 2010

- Koschatzky K., Reinhard M., Grenzmann C., Forschungs- und Entwicklungsdienstleistungen in Deutschland. Struktur und Perspektiven eines Wachstumsmarktes, Stuttgart, 2003

- Muller E., Zenker A., Héraud J.-A., «France : Innovation System and Innovation Policy », in FRIETSCH R., SCHÜLLER M. (eds), Competing for Global Innovation Leadership: Innovation Systems and Policies in the USA, Europe and Asia, Fraunhofer Institute for Systems and Innovation Research ISI, Stuttgart, 2010

- Polt W., Berger M., Boekholt P., Cremers K., Egeln J., Gassler H., Hofer R., Rammer C., avec la collaboration de DEUTEN J., GOOD B., WARTA K., « Das deutsche Forschungs- und Innovationssystem. Ein internationaler Systemvergleich zur Rolle von Wissenschaft, Interaktionen und Governance für die technologische Leistungsfähigkeit », Johanneum Research Forschungsgesellschaft mbH Wien; Technopolis Group Amsterdam und Brighton, Zentrum für Europäische Wirtschaftsforschung (ZEW, Mannheim), in Studien zum deutschen Innovationssystem, $n^{\circ} 11 / 2010$, édité en ligne par: Expertenkommission Forschung und Innovation (EFI), Berlin. Consultable sur : www.e-fi.de/fileadmin/Studien/Studien_2010/11_2010_Ful_System.pdf [consulté le 21-09-2011]

- Rammer C., Pesau A., «Innovationsverhalten der Unternehmen in Deutschland 2009. Aktuelle Entwicklungen - Bundesländerunterschiede - internationaler Vergleich », Zentrum für Europäische Wirtschaftsforschung (ZEW), Studien zum deutschen Innovationssystem, $n^{\circ} 7 / 2011$. Edité en ligne par : Expertenkommission Forschung und Innovation (EFI), Berlin. Consultable sur : www.efi.de/fileadmin/Studien/StuDIS_2011/StuDIS_7_2011.pdf [consulté le 21-09-2011]

- Statistisches Bundesamt, Statistisches Jahrbuch 2011 für die Bundesrepublik Deutschland mit Internationalen Übersichten, Wiesbaden, 2011

-StifTeRVERBAND füR Die DeUtsche Wissenschaft, Forschung \& Entwicklung. Zahlen \& Fakten aus der Wissenschaftsstatistik $\mathrm{GmbH}$ im Stifterverband, 2011. Consultable sur: www. stifterverband. org/publikationen_und_podcasts/wissenschaftsstatistik/fue_facts/fue_facts_2011-01.pdf [consulté le 21-09-2011]

- Tippmann V., ZenKer A., Muller E., « La coopération Fraunhofer-Carnot. Fer de lance de futures politiques européennes de recherche appliquée ? », in LESOURNE J., RANDET D. (eds), La Recherche et I'Innovation en France, Paris, 2010

-TRISCHLeR H., BRUch R. v., Forschung für den Markt: Geschichte der Fraunhofer-Gesellschaft, Munich, 1999

-www.fraunhofer.de

•www.fraunhofer.de/en/press/research-news/2011/december/Franco-GermanBusinessAward2011. html

-www.instituts-carnot.eu. 